\title{
Adrenomedullin in COVID-19 induced endotheliitis
}

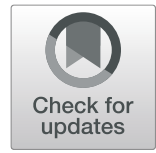

\section{Darius Cameron Wilson ${ }^{1 *}$ D, Joerg C. Schefold ${ }^{2}$, Jaume Baldirà ${ }^{3}$, Thibaud Spinetti ${ }^{2}$, Kordo Saeed ${ }^{4}$ and Gunnar Elke}

Despite the exponential growth in research following the rapid spread of SARS-CoV-2 and subsequent COVID-19 pandemic, the underlying pathophysiological mechanisms in COVID-19 patients remain poorly understood. The increased incidence of cardiovascular and thromboembolic complications, immune cell deactivation and sepsis-like multiple organ failure suggests the involvement of multiple pathways. Accordingly, recent studies have proposed that virus-induced endothelial dysfunction and damage, resulting in impaired vascular blood flow, coagulation and leakage, may partially explain the development of organ dysfunction and oedema [1]. Hence, the development of endotheliitis may be a prominent, yet partly under recognised, feature of COVID-19 induced critical illness.

Whilst numerous pro-inflammatory cytokines and blood biomarkers have already been compared in patients with different severities of COVID-19 - to date - no study, report or editorial has described the potential role of adrenomedullin (ADM) during the host response to COVID19. This is surprising, since ADM has been shown to play a key role in reducing vascular (hyper) permeability and promoting endothelial stability and integrity following severe infection [2]. Thus, ADM may also be of interest within COVID-19 induced endotheliitis. Indeed, a recent study investigating gene upregulation in patients with systemic capillary leak syndrome (SCLS), characterised by plasma leakage into peripheral tissue and transient episodes of hypotensive shock and oedema, found that ADM was not only one of the most upregulated genes, but that subsequent application to endothelial cells resulted in a protective effect on vascular barrier function [3].

Furthermore, recent clinical studies on sepsis patients upon emergency department (ED) presentation and

\footnotetext{
* Correspondence: Dwilso52@caledonian.ac.uk

'Shock, Organ Dysfunction and Resuscitation Research Group, Vall d'Hebron Institut of Research, Barcelona, Spain

Full list of author information is available at the end of the article
}

during intensive care (ICU) treatment using the stable protein surrogate, mid-regional proadrenomedullin (MR-proADM), found that its assessment could accurately identify disease progression in patients with nonsevere clinical signs and symptoms, safely increase outpatient treatment with decreased readmission rates and no subsequent mortalities [4], and identify patients requiring a rapid administration of antibiotics or triage to the ICU [5]. Despite the low number of severe viral cases within each of these studies (between 2.1\% [3] and 3.4\% [4]), similar hypotheses can also be formulated for patient populations with COVID-19.

The assessment of MR-proADM in future COVID-19 studies may therefore provide important information into the pathophysiological mechanisms underlying endotheliitis and subsequent organ dysfunction. The early identification of patients likely to develop severe clinical symptoms requiring subsequent hospitalisation, as well as the safe discharge of those already hospitalised, may be of particular importance in regions where healthcare systems are used to full capacity.

\section{Abbreviations \\ ADM: Adrenomedullin; ED: Emergency department; ICU: Intensive care unit: MR-proADM: Mid-regional proadrenomedullin; SARS-CoV-2: Severe acute respiratory syndrome coronavirus 2; SCLS: Systemic capillary leak syndrome}

\section{Acknowledgements \\ None. \\ Authors' contributions \\ DCW was the primary author and editor of the manuscript. All authors critically reviewed and approved the final manuscript. DCW takes responsibility for the paper as a whole.}

\section{Funding}

No funding was received for this communication.

\section{Availability of data and materials Not applicable}




\section{Ethics approval and consent to participate}

Not applicable

\section{Consent for publication}

Not applicable

\section{Competing interests}

All authors have provided information on potential conflicts of interests directly or indirectly related to the work submitted in the journal's disclosure forms. All authors reported no conflicts of interest.

\section{Author details}

${ }^{1}$ Shock, Organ Dysfunction and Resuscitation Research Group, Vall d'Hebron Institut of Research, Barcelona, Spain. Department of Intensive Care Medicine, Inselspital, Bern University Hospital, University of Bern, Bern, Switzerland. ${ }^{3}$ Intensive Care Department, Hospital de la Santa Creu i Sant Pau, Barcelona, Spain. ${ }^{4}$ University Hospital Southampton NHS Foundation Trust, Southampton, UK. ${ }^{5}$ Department of Anaesthesiology and Intensive Care Medicine, University Medical Center Schleswig-Holstein, Campus Kiel, Arnold-Heller-Str. 3 Haus R3, 24105 Kiel, Germany.

Received: 17 June 2020 Accepted: 3 July 2020

Published online: 09 July 2020

\section{References}

1. Varga Z, Flammer AJ, Steiger $P$, et al. Endothelial cell infection and endotheliitis in COVID-19. Lancet. 2020;395(10234):1417-8. https://doi.org/ 10.1016/S0140-6736(20)30937-5

2. Elke G, Bloos F, Wilson DC, et al. The use of mid-regional proadrenomedullin to identify disease severity and treatment response to sepsis - a secondary analysis of a large randomised controlled trial. Crit Care. 2018;22:79 https://doi.org/10.1186/s13054-018-2001-5.

3. Xie Z, Chen WS, Yin Y, et al. Adrenomedullin surges are linked to acute episodes of the systemic capillary leak syndrome (Clarkson disease). J Leukoc Biol. 2018;103(4):749-59. https://doi.org/10.1002/JLB.5A0817-324R.

4. Saeed K, Wilson DC, Bloos F, et al. The early identification of disease progression in patients with suspected infection presenting to the emergency department: a multi-centre derivation and validation study. Crit Care. 2019;23:40 https://doi.org/10.1186/s13054-019-2329-5.

5. del Castillo JG, Wilson DC, Clemente-Callejo C, et al. Biomarkers and clinical scores to identify patient populations at risk of delayed antibiotic administration or intensive care admission. Crit Care. 2019;23:335 https://doi. org/10.1186/s13054-019-2613-4.

\section{Publisher's Note}

Springer Nature remains neutral with regard to jurisdictional claims in published maps and institutional affiliations. 